\title{
Introduction: Practical Cognitive Agents and Robots
}

\author{
Xiaoping Chen · Wei Liu · Mary-Anne Williams
}

Published online: 12 March 2009

Springer Science+Business Media, LLC 2009

Currently, performing complex tasks in unstructured and dynamic environments is still a significant challenge to traditional software agents and autonomous robots. Advances in intelligent agents and robotics over the last decade have been extraordinary, promising unprecedented potential for future scientific breakthroughs and applications. At the present time, however, a number of research bottlenecks to future advancement have been identified such as the creation of cognitive agents that can make their own representations, that are aware of what they are doing, that can adapt robustly to changing conditions and requirements, and that can deal with novel and unexpected situations.

This volume contains selected papers addressing these challenging issues. The papers were originally presented at the International Symposium on Practical Cognitive Agents and Robots (PCAR2006). They were subsequently enriched and extended after the symposium and have undergone several rounds of rigorous peer reviewing. The PCAR symposium is unique and innovative in its focus, taking a contemporary integrative transdisciplinary practical view of cognitive agents and robots. This volume contains high quality research that describes practical ideas and scientific results from human-robot interaction to high-level reasoning that address the following important specific research issues:

- Life-long interaction between robots and humans

- Grounding representations in experiences

- Integrating knowledge representation and robot behaviour

- Inter-robot and human-robot interaction and collaboration

- Temporal reasoning in cognitive robotic unmanned aerial vehicles

- Robot perception, world modelling, prediction, control, planning and acting.

X. Chen

The University of Science and Technology of China, Hefei, China

W. Liu $(\bowtie)$

The University of Western Australia, Perth, Australia

e-mail: wei.vivian.liu@gmail.com

M.-A. Williams

The University of Technology, Sydney, Australia 
The first paper explores open-ended learning systems. The author Masahiro Fujita is a Chief Distinguished Researcher at Sony Corporation. His robotics research over the years has been driven by his curiosity and passion. He was the technical leader of Sony's development of the innovative four-legged robot the AIBO and the sophisticated state-of-the-art humanoid QRIO. His work has led to several groundbreaking scientific ideas in the important field of robotics, and as a result he has opened up a whole new world of exciting research with high impact practical outcomes. In addition, he was instrumental in starting the Sony Legged League at RoboCup in 1998 and his vision of the future continues to inspire. In his paper, Fujita proposes a new approach to developing intelligent systems called Intelligence Dynamics. His models of learning have been implemented in real state-of-the-art robots including the humanoid QRIO. His interactivity and learning approach is based on three key pillars: embodiment, system dynamics and a constructive methodology. The paper provides an architecture for Intelligence Dynamics whose prediction and control functions emphasize links with evolution and development. It contributes significantly to the concept of open-ended learning systems and it demonstrates that a robot can learn how to build a tower of blocks on the fly from a human teacher.

The second paper by Williams, McCarthy, Gärdenfors, Stanton and Karol introduces a novel framework for inspecting, analyzing and designing grounding capabilities in agents and robots. Intelligent agents require high level representations and the grounding framework presented helps to explore the grounding capabilities of existing agents and to guide the development of new ones. The framework is based on ten clearly articulated principles which state the key underlying assumptions upon which the framework is founded such as the novel idea that the groundedness of agents can be graded and multidimensional. In essence the framework provides a template for analysis and comparison of systems, which produces a comprehensive and focused account of grounding capabilities. Two real world applications of the framework are briefly described for the purpose of illustration. The first provides a simple analysis of an existing system and the second a comparison of two different systems.

The third paper by Brenner and Nebel provides a new, principled and powerful approach to the interleaving of acting and sensing so that planning is continual. This approach informs an agent when to switch between planning and acting. It provides an algorithm using the planning language MAPL that enables agents to deliberately postpone parts of their planning process and to actively discover missing relevant information that can be used to improve the plan. The continually planning algorithm is tested in a simulation environment and these tests provide a proof-of-concept that demonstrates that deliberate action planning can be used efficiently even in complex multi-agent environments.

The fourth and final paper by Doherty, Kvarnström and Heintz develops a planning and execution framework that supports temporal reasoning. The authors from the new Future Aviation Systems Center, a joint venture between Saab Aero Systems and Linköping University, are international leaders in the areas of automated planning and unmanned aerial vehicle research. They use Temporal Action Logic, which is a logic for reasoning about action and change, to generate mission plans. The resulting system is deployed on a rotorbased unmanned aircraft system which relies on the timely collection and abstraction of sensor data in real time while flying. The system was tested using a challenging emergency services scenario involving the identification of injured civilians on the ground and logistics missions that deliver medical and food supplies.

The editors would like to thank the National Natural Science Foundation of China, the Australia Federal Governement Department of Education Science and Technology, the Australian Research Council, the University of Science and Technology of China, the University 
of Technology, Sydney, and the University of Western Australia who all generously funded the PCAR symposium. We would like to express our gratitude to the PCAR 2006 Program Committee who acted as reviewers and an additional reviewer, Sylvie Thiebaux who provided additional expertise in the area of planning. 